\title{
Exhaustive Running Exercise Induce Tyrosine Phosphorylation of Band 3 in Rat Erythrocytes
}

\author{
Yanlian Xiong Yaojin Li Yanlei Xiong Yajin Zhao Fuzhou Tang Xiang Wang \\ Key Laboratory of Biorheological Science and Technology, Ministry of Education, College of \\ Bioengineering, Chongqing University, Chongqing, P.R. China
}

\section{Key Words}

Exhaustive exercise $\cdot$ Phosphorylation $\bullet$ Erythrocyte band $3 \cdot$ Oxidative stress

\begin{abstract}
Background/Aims: In vitro studies have shown that band-3 function is mainly regulated by its phosphorylation status. The main purpose of the study was to investigate whether band-3 phosphorylation status interferes with an exhaustive running exercise-related dysfunction of RBC deformability. Methods: Rats were divided into sedentary control (C) and exercise test (ET) groups. The ET group was divided further into exhaustive running exercise (ERE) and moderate running exercise (MRE) subgroups. Results: Tyrosine phosphorylation of band-3 was significantly elevated in the absence of reducing agent, consistent with the emergence of band-3 clustering in the ERE group compared with the control and MRE groups. The elongation index (EI) was found to decline significantly in the ERE group compared with the $C$ and MRE groups under shear stress (control group, $0.41 \pm 0.01$ at $3 \mathrm{~Pa}$ and $0.571 \pm 0.008$ at $30 \mathrm{~Pa}$; ERE group, $0.3140 \pm 0.013$ at $3 \mathrm{~Pa}$ and $0.534 \pm 0.009$ at $30 \mathrm{~Pa} ; \mathrm{P}<0.001$ and $\mathrm{P}<0.002$, respectively). Conclusion: Our results suggest that exhaustive running exercise results in elevated band-3 tyrosine phosphorylation and alters band-3 membrane organization. Furthermore, it appears that exhaustive running exercise induced band 3 phosphorylation is due to the oxidation of critical sulfydryl groups of a membrane phosphatase (PTP).
\end{abstract}

Copyright $\odot 2013$ S. Karger AG, Basel

\section{Introduction}

During the past three decades, our knowledge of the biological implications of exerciseinduced oxidative stress has expanded [1-3]. Several studies have suggested that damage to erythrocytes occurs during physical exercise [4-6]. Many hypotheses have been proposed to explain such exercise-induced erythrocyte injury. However, the exact mechanisms leading to 
exercise-induced erythrocyte dysfunction are not known. A recent investigation by Senturk et al. demonstrated that exhaustive exercise resulted in elevation of oxidant stress and deterioration of erythrocyte structure [7]. Mao et al. showed that hypoxic exercise training causes erythrocyte senescence and rheological dysfunction because of depressed Gardos channel activity [8]. However, whether erythrocyte band-3 (SLC4A1; EB3) is attacked by reactive oxygen species (ROS) or if its function is altered during exhaustive exercise in vivo is not known.

Band-3 is the major integral protein of the RBC membrane, and it plays a crucial role in membrane functional organization, stability and RBC volume regulation [9-11]. In contrast, changes in band-3 structure and its membrane organization have been observed after oxidative stress or during physiological aging of RBCs and are accompanied by cell shrinkage, decreased cell deformability and alterations in RBC metabolic activity [12]. Thus, band-3 is a potential molecular target in exercise-related blood disease, and alterations in band-3 may be associated with RBC dysfunction during exercise conditions.

In vitro studies have shown that band-3 function is mainly regulated by its phosphorylation status [13-15]. Band-3 phosphorylation is promoted by protein tyrosinekinases (PTKs; e.g., syk and lyn) and dephosphorylation is performed by protein tyrosinephosphatases (PTPs) [16-19]. Zipser et al. previously identified a PTP associated with band 3 in the human erythrocyte membrane, which is normally highly active and prevents the accumulation of band 3 phosphotyrosine [20]. Increased band-3 tyrosine phosphorylation has been shown to stimulate glycolysis and alter its anion transport activity [21,22]. Studies have also shown that elevated band-3 phosphorylation alters cytoskeletal organization by modulating the interactions between band-3 and cytoskeletal anchoring proteins [23, 24].

Based on these observations, we hypothesized that the RBC dysfunction observed during exercise-induced oxidative stress could be associated with alterations in the conformation or phosphorylation of Band 3, which in turn leads to dysfunction of RBC deformability. To test this hypothesis, we assessed the exact relationship between exercise-induced oxidative stress in RBCs and the EB3 phosphorylation status during running exercise. Furthermore, we investigated the effects of EB3 phosphorylation on erythrocyte deformability during exercise-induced oxidative stress.

\section{Materials and Methods}

\section{Animal care}

Animals used in this study were maintained in accordance with the Guide for the Care and Use of Laboratory Animals published by the US National Institutes of Health (NIH publication no. 85-23, revised 1996), and all procedures were approved by the Institutional Review Board of the Institute of Health Sciences, Chongqing Institutes for Biological Sciences, Chinese Academy of Sciences and Chongqing University School of Medicine (Chongqing, China).

\section{Animals, exercise protocol and blood samples}

Adult male Wistar rats ( 8 weeks, 200-250 g) were used. Animals were reared at room temperature $\left(22 \pm 3^{\circ} \mathrm{C}\right)$, relative humidity of $40-60 \%$ and an illumination time from 07:30 to 20:00. The rats were divided randomly into three groups of 10 . The rats were initially divided into two groups: sedentary control (C) and exercise test (ET) group. The ET group was then divided further into two subgroups: exhaustive running exercise (ERE) and moderate running exercise (MRE). ET group rats were exercised according to a modification of the protocol designed by Davies et al. [25]. Briefly, all running exercise groups were introduced to running on a motor-driven rodent treadmill. The treadmill was equipped with an electric shocking grid on the rear barrier to provide exercise motivation to the animals. Animals in the ERE group started treadmill running at a speed of $25 \mathrm{~m} / \mathrm{min}$ speed with a $5 \%$ gradient, and reached a $15 \%$ gradient in $20 \mathrm{~min}$. Running was continued until exhaustion, and rats were protect from dehydration through intermittent drinking during exhaustive exercise. Exhaustion was defined as the inability of a rat to right itself if laid on its side. Animals in the MRE group started treadmill running at a speed of $25 \mathrm{~m} / \mathrm{min}$ 
Xiong et al.: Band-3 Phosphorylation in Exhaustive Running Exercise

with a $5 \%$ gradient, and reached a $15 \%$ gradient in $20 \mathrm{~min}$. Each rat ran for $40 \mathrm{~min}$. The sedentary group rats were placed on the treadmill but remained sedentary for the same period of time as the exercisetrained rats.

Rats were anesthetized $(50 \mathrm{mg} / \mathrm{kg}$ sodium pentobarbital, i.p.) immediately after exercise. Blood samples were obtained from the abdominal aorta of the rats under light ether anesthesia and were treated with the anticoagulant, heparin sodium $(15 \mathrm{U} / \mathrm{ml})$. After humanely sacrificing the animals by ether anaesthetization, the rats were further dissected and the heart blotted with clean tissue paper and then weighed. The organ-to-body weight ratios were determined according to the equation described by Yakubu et al. [26].

\section{Preparation of RBC suspensions and ghosts}

Fresh heparinized blood was centrifuged at $900 \times \mathrm{g}$ at $4^{\circ} \mathrm{C}$ for $10 \mathrm{~min}$. Plasma and the buffy coat was removed. After removal of plasma, cells were filtered through cellulose to remove leucocytes and platelets, as described in [27]. Packed RBCs were washed thrice with phosphate-buffered saline (PBS) containing $5.5 \mathrm{mM}$ glucose and $0.08 \%$ bovine serum albumin (BSA). At each washing step, the upper one-fifth volume of the packed RBCs was removed. The final RBC pellet was resuspended to a final hematocrit of $10 \%$ and used for experiments assessing whole RBC responses. Hematological parameters were evaluated on a Bayer Technicon Analyser ADVIA. Hematocrit and hemoglobin were manually determined [28-30].The serum EPO concentrations were determined by use of an automated solid-phase chemiluminescent immunoassay (Diagnos-tic Products, Los Angeles, CA).

For the preparation of RBC ghosts, washed packed RBCs were lysed with ice-cold $5 \mathrm{mM}$ sodium phosphate buffer ( $\mathrm{pH}$ 8.0) containing $1 \mathrm{mM}$ ethylene glycol tetraacetic acid (EGTA), 1 mM sodium orthovanadate, and $1 \mathrm{mM}$ phenylmethylsulfonyl fluoride (lysis buffer) and incubated at $4^{\circ} \mathrm{C}$ for $10 \mathrm{~min}$. RBC ghosts were washed thrice by centrifugation $\left(13,500 \mathrm{rpm}\right.$ for $20 \mathrm{~min}$ at $\left.4^{\circ} \mathrm{C}\right)$ to obtain "white" ghosts.

\section{Antioxidant and Oxidant Stress Parameters}

Antioxidant status: GSH levels were determined using the method described by Dringen and Hamprecht [31]. The activities of SOD were assessed using the methods of Fridovich [32].

Thiobarbituric acid-reactive substances (TBARs): The extent of lipid peroxidation of RBC membranes was estimated by measuring TBARS levels according to the method of Stocks and Dormandy [33]. TBARS levels were estimated by measuring absorbance at $532 \mathrm{~nm}$ after a reaction with thiobarbituric acid. Trichloroacetic-acid extracts of RBC samples were used to circumvent the interference of proteins with TBARS determinations. Results were expressed as nanomoles per gram hemoglobin (Hb).

\section{Determination of free thiol groups in erythrocyte membranes}

SH-groups of erythrocyte membrane proteins were quantified according to Anderson [34] and Yamaguchi [35]. Like the reduced glutathione hormone (GSH) molecules, the thiol groups in erythrocyte membrane proteins react with 5, 59-dithiobis (2-nitrobenzoic acid) (DTNB) to form a product possessing an absorbance at $415 \mathrm{~nm}$, which is then used to analyze the free thiol group contents. In this experiment, erythrocyte membranes were solubilized by a solution containing $0.3 \mathrm{ml}$ of $10 \%$ sodium dodecyl sulphate (SDS) and $4 \mathrm{ml}$ of $10 \mathrm{mM}$ PBS buffer. Then $0.1 \mathrm{ml}$ of $10 \mathrm{mM}$ DTNB in $10 \mathrm{mM}$ PBS was added. Based on the absorbance at $415 \mathrm{~nm}$ after incubation at $37^{\circ} \mathrm{C}$ in water bath for $15 \mathrm{~min}$, the contents of SH-groups were determined using GSH as a standard.

\section{Electrophoresis and immunoblotting analyses of plasma membranes}

Erythrocytes were suspended to $10 \%$ hematocrit in $25 \mathrm{mM}$ HEPES buffer, $\mathrm{pH} 7.3 / 125 \mathrm{mM} \mathrm{NaCl}$ and incubated for $30 \mathrm{~min}$ at $37^{\circ} \mathrm{C}$ in the presence or absence of $5.0 \mathrm{mM}$ dithiothreitol (DTT). The protein content of the membranes was quantified using the detergent compatible (DC) protein assay and was solubilized in Laemmli buffer under non-reducing or reducing conditions (i.e. in the absence and presence of $10 \mathrm{mM}$ DTT) at a volume ratio of 1:1. Sodium dodecyl sulfate-polyacrylamide gel electrophoresis (SDS-PAGE) was conducted by heating the samples for $8 \mathrm{~min}$ at $100^{\circ} \mathrm{C}$ and loading $10 \mu \mathrm{g}$ of membrane proteins on a $5-15 \%$ linear acrylamide gradient gel (10 $\mu$ g protein/lane) according to Laemmli for protein staining by colloidal Coomassie Blue. 
Xiong et al.: Band-3 Phosphorylation in Exhaustive Running Exercise

For western blot analyses, $30 \mu$ g of protein was loaded in each lane, transferred onto polyvinylidene fluoride membranes, immunostained with primary antibodies (rabbit polyclonal to EB3; diluted 1:2,000; Abcam, Cambridge, UK) or (goat polyclonal to phosphotyrosine; diluted 1:2,000; Abcam, Cambridge, UK), using anti-rabbit and anti-goat peroxidase-labeled secondary antibodies, detected by chemiluminescence according to the manufacturer's protocol (Pierce Biotechnology, Rockford, IL, USA). Quantification of Coomassie blue-stained gels and electrochemiluminescence-developed immunoblots was conducted by lengthwise scanning densitometry using a gel analyzer image-processing program (ver1.0, Biosure, Athens, Greece).

Immunofluorescence and image analyses

RBCs were fixed with $4 \%$ paraformaldehyde and $0.05 \%$ glutaraldehyde in PBS and permeabilized in the same solution containing $0.05 \%$ Triton X-100. After being blocked with 3\% BSA and $0.1 \%$ Tween 20 in PBS to block non-specific protein binding, cells were treated with primary antibodies (rabbit polyclonal to EB3; Abcam) diluted in $10 \mathrm{mg} / \mathrm{mL}$ PBS for $1 \mathrm{~h}$ at room temperature. The sources of all antibodies were as mentioned above. Cells were washed at 3-min intervals thrice with gentle shaking, and then incubated for $1 \mathrm{~h}$ with secondary antibodies (anti-rabbit IgG; Santa Cruz Biotechnology, Santa Cruz, CA, USA) at 1:700 dilution in PBS, and washed thrice in PBS. Fluorescence was imaged using an Olympus IX71 microscope with a 63/1. 25 oil immersion objective and equipped with a CCD camera (Olympus, Tokyo, Japan).

\section{Determination of PTP activity and PTP analysis by immunoblotting}

PTP activity was estimated by using p-nitrophenyl phosphate disodium salt (p-NPP) as substrate according to published procedures, with some modifications. Erythrocyte white membranes were suspended in buffer containing $15 \mathrm{mM}$ p-NPP and incubated at $37{ }^{\circ} \mathrm{C}$ for $30 \mathrm{~min}$ in the absence or presence of $5 \mathrm{mM}$ dithiotreitol (DTT). The reaction was terminated by addition of $0.1 \mathrm{M} \mathrm{NaOH}$, the samples were centrifuged (9000 g), and the release of p-nitrophenol from p-NPP was measured spectroscopically at $410 \mathrm{~nm}$.

\section{Erythrocyte deformability}

RBC deformability was determined by laser diffraction analyses using an ektacytometer (LBY-BX; Precil, Beijing, China). Briefly, before measurement, the ektacytometer was preheated for 30 min to let the internal temperature reach $37^{\circ} \mathrm{C}$. A low-hematocrit suspension of RBCs in $1 \mathrm{~mL}$ polyvinyl pyrrolidone (mol wt 360,000; Sigma, St. Louis, MO) solution (15\% in PBS; solution reparation, 15 g PVP, 284 mg Na2HPO4, $68 \mathrm{mg} \mathrm{KH} 2 \mathrm{PO} 4,0.38 \mathrm{~g} \mathrm{NaCl}$, add water (80 mL) until completely dissolved, adjust the pH to 7.4 using $10 \% \mathrm{NaOH}$; complement water to $100 \mathrm{~mL}$ ) was sheared in a Couette system composed of a glass cup and a precisely fitting bob with a gap of $0.3 \mathrm{~mm}$ between the cylinders. A laser beam was directed through the sheared sample. The diffraction pattern produced by the deformed cells was analyzed by a computer attached to the ektacytometer. Based on the geometry of the elliptical diffraction pattern, the elongation index (EI) was calculated under shear stresses $0.3 \mathrm{~Pa}$ and $30 \mathrm{~Pa}$ as:

$$
\mathrm{EI}=(\mathrm{L}-\mathrm{W}) /(\mathrm{L}+\mathrm{W})
$$

where $\mathrm{L}$ and $\mathrm{W}$ are the length and width of the diffraction pattern, respectively. An increased EI at the given shear stress indicates greater cell deformation and hence greater RBC deformability. For studies using pervanadate, cell suspensions were pre-incubated with pervanadate ( $2 \mathrm{Mm}$, Beyotime, Shanghai, China) or vehicle for $10 \mathrm{~min}$ at $25^{\circ} \mathrm{C}$ prior to testing $\mathrm{RBC}$ for cell deformability or anion-transport activity.

\section{Statistical analyses}

Statistical analyses of the data were performed by using statistics software packages (Origin 8.5 Pro, Northampton, USA and GraphPad Prism 4, La Jolla, USA). Data were analyzed by multi-way analysis of variances (ANOVA). Descriptive statistics of the data is presented as mean6 standard error of means (S.E.M.) unless described otherwise. Statistical differences were considered to be significant for values of $\mathrm{P}<0.05$.

\section{Results}

At the end of exercise, there were no significant differences in body weight and heart weight among groups. Training also did not alter the hematocrit and total blood $\mathrm{Hb}$ values 
Table 1. Body weight, heart weight, exhaustion time, Haematological quantities and erythropoietic activity of control and exercise trainings groups

\begin{tabular}{lccccc}
\hline & C & \multicolumn{2}{c}{ MRE } & \multicolumn{2}{c}{ ERE } \\
& & Pre & Post & Pre & Post \\
\hline Body weight, g & $261 \pm 9.2$ & $261 \pm 8.5$ & $256 \pm 5.6$ & $257 \pm 7.9$ & $243 \pm 6.8$ \\
Heart weight, g & $0.871 \pm 0.031$ & $0.871 \pm 0.045$ & $0.863 \pm 0.073$ & $0.871 \pm 0.042$ & $0.863 \pm 0.052$ \\
Exhuastion time, min & 0 & 0 & 40 & 0 & $243 \pm 9.6^{* *}$ \\
Hematocrit, \% & $40.1 \pm 0.6$ & $39.8 \pm 0.6$ & $39.2 \pm 0.4$ & $40.2 \pm 0.6$ & $39.6 \pm 0.3$ \\
Hemogiobin, g/dl & $12.3 \pm 0.3$ & $12.1 \pm 0.3$ & $12.4 \pm 0.3$ & $12.3 \pm 0.3$ & $12.1 \pm 0.3$ \\
EPO, mU/ml & $12.8 \pm 0.6$ & $12.6 \pm 0.5$ & $12.9 \pm 0.7$ & $12.8 \pm 0.6$ & $13.2 \pm 0.5$ \\
RDW (\%) & $12.2 \pm 0.3$ & $12.2 \pm 0.3$ & $12.3 \pm 0.6$ & $12.2 \pm 0.3$ & $13.5 \pm 0.3^{*} \dagger$ \\
Reticulocytes (\%) & $2.20 \pm 0.70$ & $2.20 \pm 0.70$ & $2.32 \pm 0.51$ & $2.20 \pm 0.70$ & $2.36 \pm 0.65$ \\
\hline
\end{tabular}

EPO, serum erythropoietin concentration; RDW, red cell distribution width; Values are means \pm SD. C, control groups; MRE, moderate running exercise; ERE, exhaustive running exercise. Pre, pretraining; Post, posttraining. ${ }^{*} \mathrm{P}<0.05$ and ${ }^{* *} \mathrm{P}<0.01$, Pre vs. Post; $\nmid \mathrm{P}<0.05$, ERE vs. C.

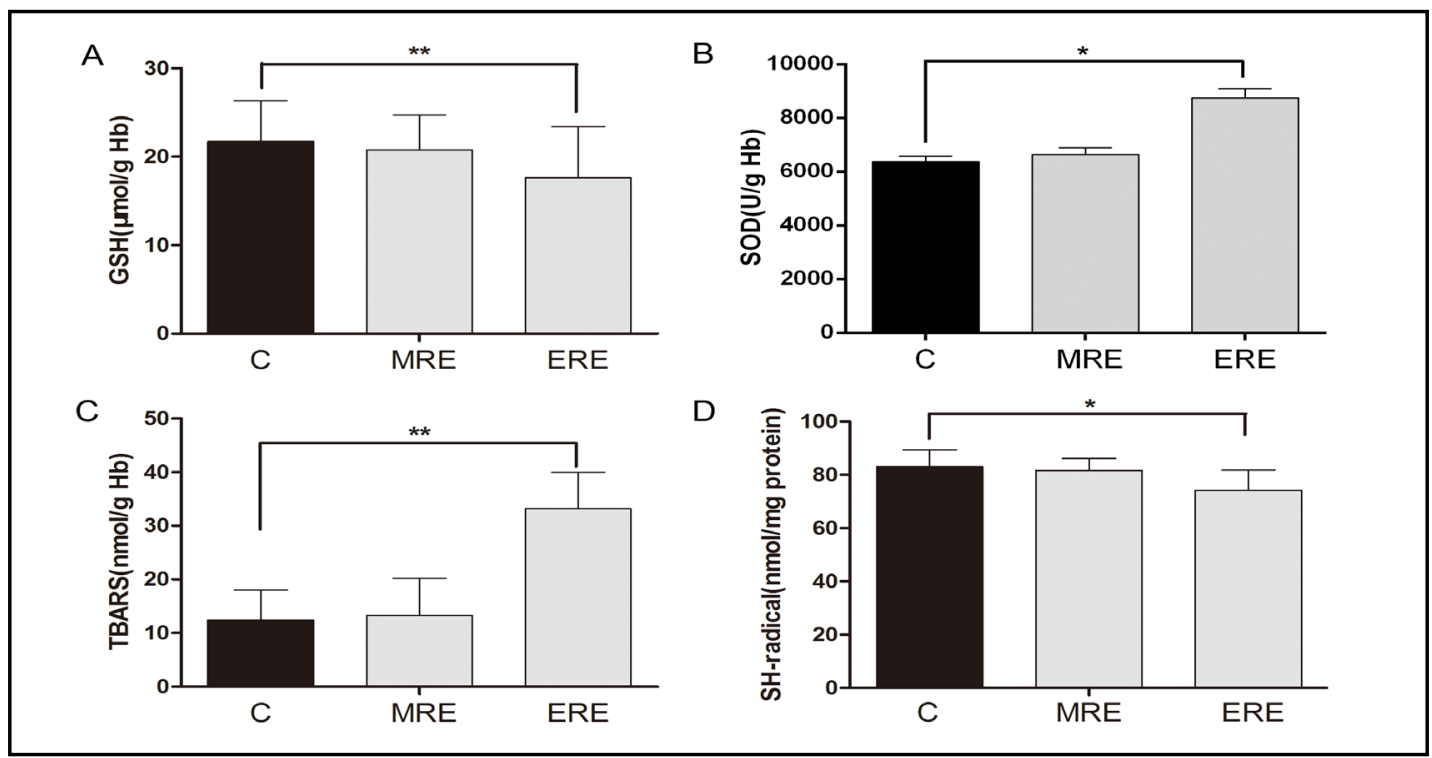

Fig. 1. Erythrocyte antioxidant and oxidant stress parameters. The levels of GSH (A),SOD (B), TBARS (C) and thiol radicals (D) were determined in erythrocytes collected from exercised and control animals. Data are expressed as means \pm SEM with the significance level set at $* \mathrm{P}<0.05$ and ${ }^{* *} \mathrm{P}<0.01$ for the exercise group compared with the corresponding control group.

(Table 1). Immediately after the exhaustive running exercise there was a non-significant increase in reticulocytes count and serum EPO levels. Significantly increase in red cell distribution width (RDW) was found after exhaustive running exercise. In contrast, there were no significant changes in RDW in RBC collected from control and MRE groups.

\section{Antioxidant status and oxidant stress parameters in erythrocytes}

To determine the impact of different exercises on RBC antioxidant status and oxidant stress parameters, TBARS and GSH were analyzed and the results are presented in Fig. 1ABC. TBARS levels were significantly increased in exhaustive running exercise animals compared with the $\mathrm{C}$ group (C group, $12.43 \pm 5.67 \mathrm{nmol} / \mathrm{g} \mathrm{Hb}$; ERE group, $33.25 \pm 6.45 \mathrm{nmol} / \mathrm{g} \mathrm{Hb}$; P $<0.05)$. There were significant differences in GSH levels between the C group $(21.78 \pm 4.55$ $\mu \mathrm{mol} / \mathrm{g} \mathrm{Hb})$ and the ERE group (17.69 $\pm 5.66 \mu \mathrm{mol} / \mathrm{g} \mathrm{Hb})$. Besides, there were significant differences between the $\mathrm{C}$ group (SOD activity, $6380 \pm 196 \mathrm{U} / \mathrm{g} \mathrm{Hb}$ ) and the ERE group (SOD activity, $8740 \pm 351 \mathrm{U} / \mathrm{g} \mathrm{Hb}$ ) with regard to the activity of SOD activity. However, TBARS, SOD 
Fig. 2. Electrophoretic and microscopic analyses of EB3 protein modifications in erythrocytes collected from exercised and control animals. (A) SDS-PAGE analyses of erythrocyte membrane proteins. (B) Fluorescent micrographs for control and exercised RBCs after immunostaining with monoclonal antibodies to EB3. Bars $=3 \mu \mathrm{m}$. The arrows indicate protein clusters after exercise-induced oxidative stress.

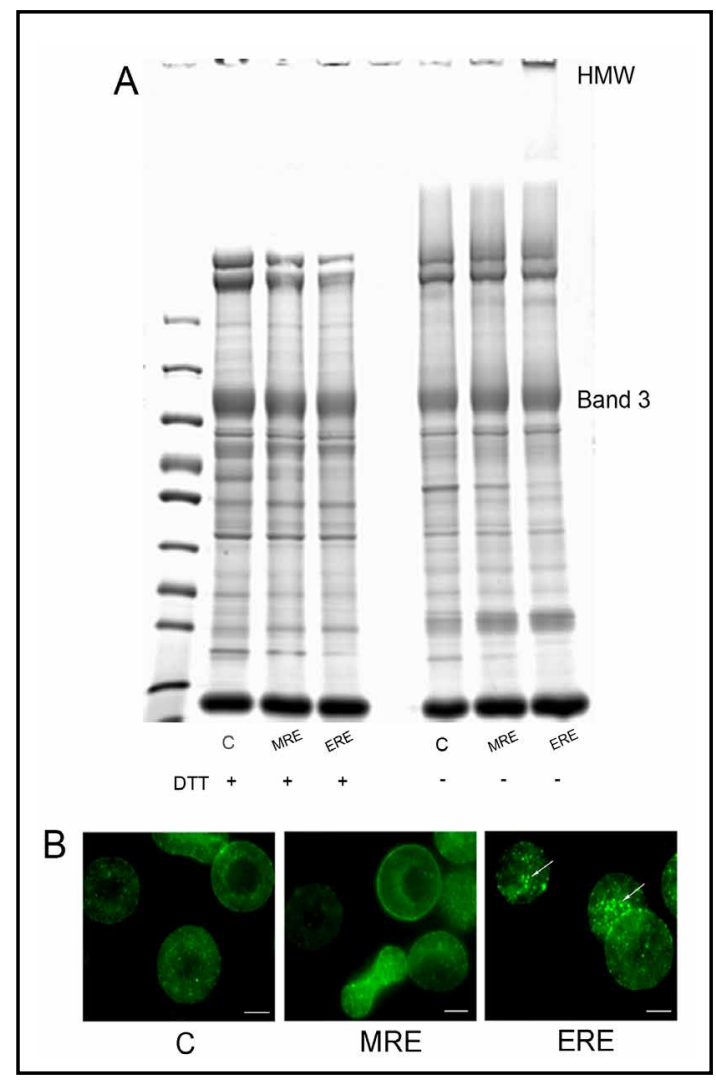

and GSH levels were not altered significantly in the MRE group compared with the C group. Fig. 1D indicates the significant decrease in free thiol groups in erythrocyte membranes of the ERE group compared with the MRE and C groups. These results indicate that red blood cell oxidative stress was induced in ERE animals but not in MRE animals.

\section{Oxidant stress-related band-3 crosslinking and altered tyrosine phosphorylation status in} the different groups

SDS-PAGE analyses of erythrocyte membrane proteins presented in Fig. 2A show that a new band of high-molecular-weight (HMW) proteins appeared above $\alpha$ and $\beta$ spectrin in the ERE group. EB3 immunoblotting is shown in Fig. 3A. Densitometric analyses showed differences in the EB3 protein at $90-100 \mathrm{kDa}$ and its clustering products at $>250 \mathrm{kDa}$ (clustered EB3) between the $C$ group and ERE group under non-reducing conditions. Band-3 was partially oxidized to HMW fractions in ERE group cells (Fig. 3A, lane 6) and was reduced back to the $95 \mathrm{kDa}$ band in erythrocytes after incubation with DTT. Subsequent microscopic analysis of band-3 (Fig. 2B) verified the presence of band-3 aggregates in cells formed after exhaustive running exercise.

The basal level of protein tyrosine phosphorylation in normal human RBCs is usually very low, making it difficult to determine the phosphorylation level by immunoblotting with anti-phosphotyrosine Ab. However, as shown in Fig. 3C, the level of tyrosine phosphorylation of band-3 was significantly elevated in the absence of reducing agent treatment $(\mathrm{P}<0.05)$. Interestingly, this result is consistent with the emergence of band-3 clustering. As shown in Fig. 3B, phosphotyrosine was observed in the erythrocytes of ET group animals, in the band corresponding to band-3 protein and in the HMW bands (Fig. 3B, lane 6). When cells were incubated with DTT, the phosphotyrosines were reduced and the protein profile reverted to the original for all three test group animals. Furthermore, the exhaustive running exercise caused an elevation of band-3 phosphorylation of about 3-fold compared with moderate running exercise. 


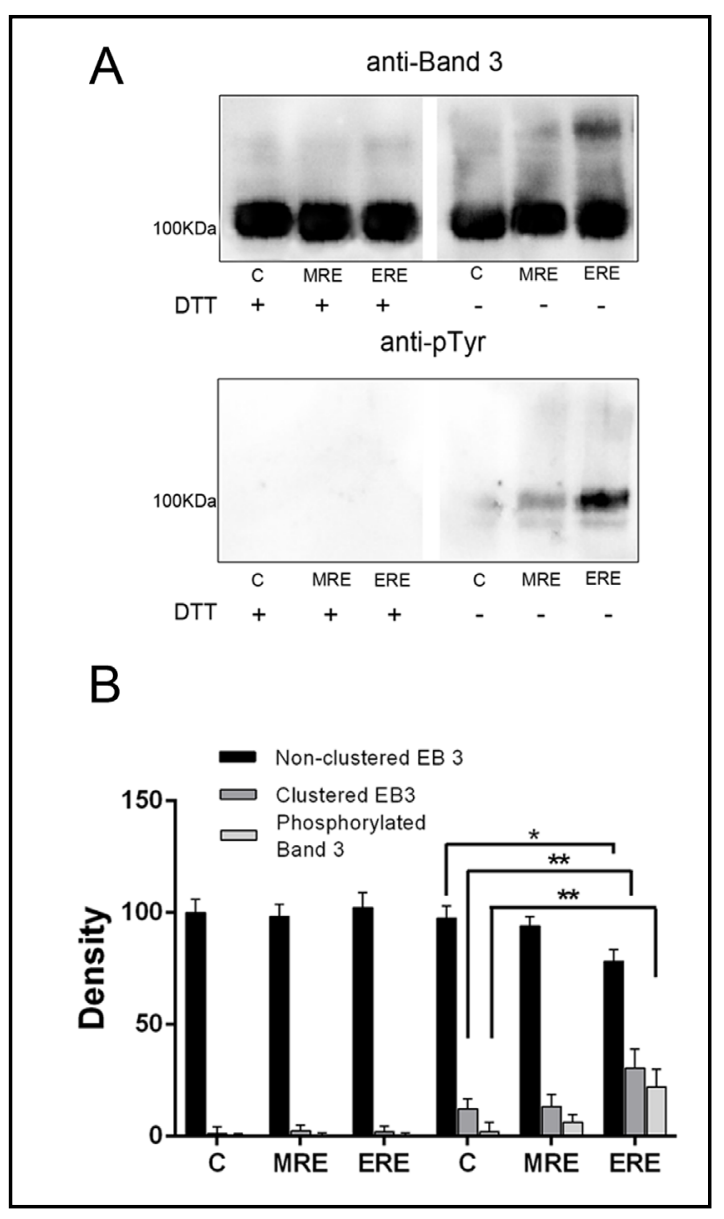

Fig. 3. Band-3 and phosphotyrosine in erythrocytes from exercised and control animals. (A) Red cells from control and exercised groups were incubated in the absence (lanes 1-3) or presence (lanes 4-6) of DTT. (B) Densitometric analyses of immunoblots of RBC ghosts (insets) from control and exercised rats probed for EB3 and phosphotyrosine. The data are the relative proportion (\%) of each protein to the total membrane proteins normalized to the controls. Error bars demonstrate the SD among donors ( $\mathrm{n}=$ 10 for control and exercised rats, respectively). Significant difference from the corresponding $\mathrm{C}$ group, $* \mathrm{P}<0.05$ and ${ }^{* *} \mathrm{P}<0.01$.

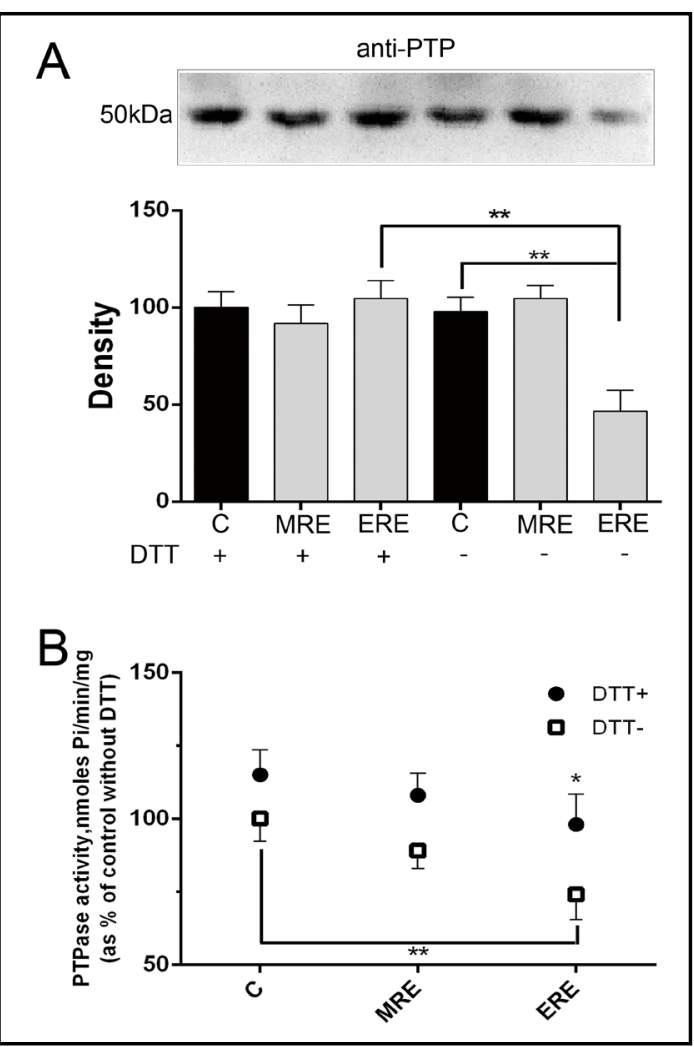

Fig. 4. PTP immunoblot and activity in ghost membranes from RBCs of different exercised and control animals. (A) Densitometric analyses of immunoblots of RBC ghosts (insets) from control and exercised rats probed for PTP. The data are the relative proportion (\%) of each protein to the total membrane proteins normalized to the controls. (B) The p-nitrophenylphosphate -hydrolysing activity of ghost membranes was measured in the presence or absence of DTT. The measured values were referred to total protein content of the samples. Finally, the calculated value of the control groups (C) (in the absence of DTT) was taken as the $100 \%$ to which all other values were referred. Error bars demonstrate the SD among donors ( $n=10$ for control and exercised rats, respectively). Significant difference from the corresponding $\mathrm{C}$ group, $* \mathrm{P}<0.05$ and ${ }^{* *} \mathrm{P}<0.01$.

\section{PTP activity of erythrocyte membranes}

Under exhaustive exercise training conditions, PTP was decreases in RBCs collected from ERE groups compared with control groups. Immunoblotting for PTP showed that in the presence of DTT, PTP back to control levels in ERE groups (As shown in Fig. 4A). The results indicate that PTP thiol oxidation causes inhibition of phosphotyrosine dephosphorylation during exhaustive exercise training and that such an inhibition can be reversed and dephosphorylation achieved by reduction of the oxidant stress-induced disulfides.

Total PTP activity was assayed in white ghost membranes. As reported in Fig. 4B, the PTP activity decreases significantly in RBCs collected from ERE groups compared with control 


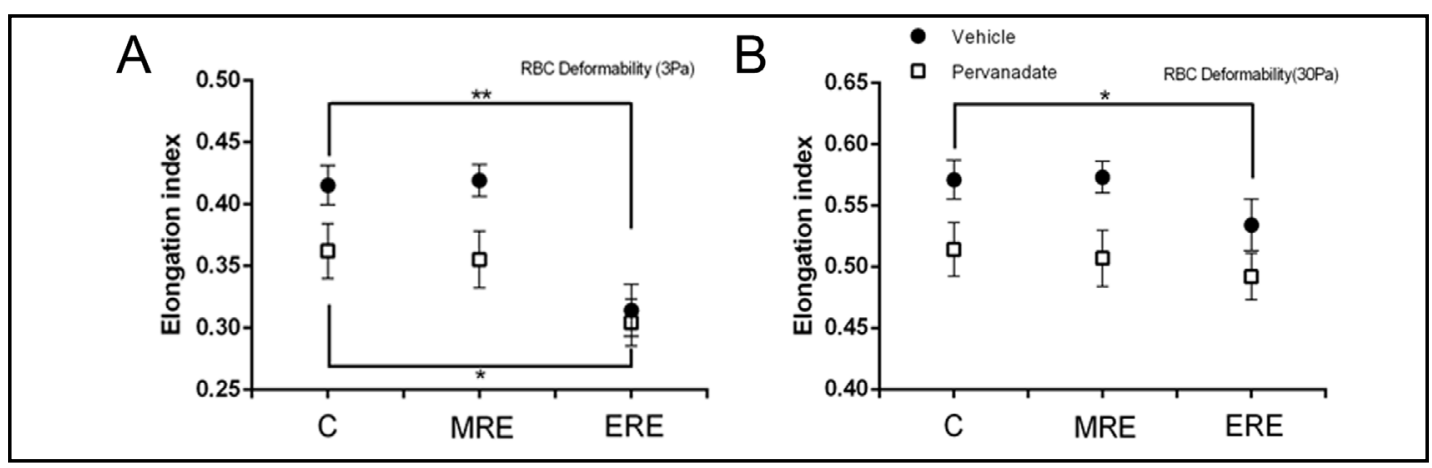

Fig. 5. Effects of exercise training on RBC deformability. RBC deformability as reflected by the elongation index in control and exercised rats tested under shear stress of $3 \mathrm{~Pa}(\mathrm{~A})$ and $30 \mathrm{~Pa}$ (B). Mean \pm SEM, from 10 independent assays, significant difference compared with vehicle. Significant difference from the corresponding $\mathrm{C}$ group, ${ }^{*} \mathrm{P}<0.05$ and ${ }^{*} \mathrm{P}<0.01$.

groups. A parallel assay was conducted under reducing conditions (DTT added) to reverse a possible partial damage of the oxidation-sensitive PTPs. As shown in Fig. 4B, the reducing treatment increases the PTP activity in all groups. The maximum extent of the recovery is appeared in ERE groups $(\mathrm{P}<0.05)$.

Effects of exercise training-induced dysfunction on RBC deformability

Erythrocytes lack nuclei and other organelles and do not perform protein synthesis. Hence, alterations in pre-existing proteins could result in recognizable changes at the membrane surface or the activity of functional proteins. To assess the impact of the tyrosine phosphorylation status on cell deformability, RBC deformability (i.e., the EI) for the C and ET groups was measured at shear stresses of $3 \mathrm{~Pa}$ and $30 \mathrm{~Pa}$ (Fig. 5). As shown in Fig. 4C and $D$, the EI was found to decline significantly in the ERE groups compared with the $C$ and MRE groups under shear stresses (control group, $0.41 \pm 0.01$ at $3 \mathrm{~Pa}$ and $0.571 \pm 0.008$ at 30 $\mathrm{Pa}$; ERE group, $0.3140 \pm 0.013$ at $3 \mathrm{~Pa}$ and $0.534 \pm 0.009$ at $30 \mathrm{~Pa}$; $\mathrm{P}<0.001$ and $\mathrm{P}<0.002$, respectively).

RBCs from C and ET group animals were isolated and incubated with pervanadate, which increases tyrosine phosphorylation by inhibiting tyrosine-phosphatases. As shown in Fig. 5B, excess pervanadate treatment resulted in a marked decline in RBC deformability in both $\mathrm{C}$ and ET group animals. These in vitro observations suggested an association between elevated band-3 tyrosine phosphorylation and decreased RBC deformability.

\section{Discussion}

In the present study, we report that exhaustive exercise training results in elevated band-3 tyrosine phosphorylation in circulating RBCs. Based on in vitro observations, tyrosine phosphorylation has been implicated in the regulation of RBC deformability. Therefore, our observation suggests that increased band-3 tyrosine phosphorylation may represent one of the underlying mechanisms contributing to RBC dysfunction during exhaustive exercise conditions. These experimental findings reflect an underlying molecular mechanism by which inadequate supplies of blood and oxygen to tissues leads to exercise-related diseases $[36,37]$.

In vivo and in vitro studies have shown that erythrocyte band 3 phosphorylation state was influenced by a variety of physiological and biochemical conditions. Deoxygenation [38], aging [39], oxidative stress [40] and shrinkage [15] et al. are found been related with the elevated phosphorylation of band 3. Previous observations also suggested that exhaustive exercise in sedentary rats induced oxidant stress injury and deterioration of erythrocyte structure [7]. In the present study, we observed elevated phosphorylation of 
oxidized/oligomeric band-3 accompanied with the appearance of oxidative damage in RBCs collected from exhaustive exercise animals. Our results further demonstrate the formation of conjugated membrane proteins to form HMW protein complexes during exhaustive exercise. Furthermore, the significant decrease of HMW bands along with decline in clustered Band-3 after incubation with reducing agents in ERE groups suggested that through the generation of reactive oxygen species (ROS) oxidative stress leading to damage of free thiol groups of the membrane proteins during exhaustive exercise, thereby inducing the oxidation of the band3 protein through the formation of reducible inter- and/or intra molecular disulfide bonds. The investigation of band-3 tyrosine phosphorylation under non-reducing and reducing conditions verified this increase in oxidative stress-induced tyrosine phosphorylation during exhaustive exercise training. Although it is widely accepted that blood cells are subjected to oxidative stress during physical exercise, oxidative stress-induced tyrosine phosphorylation has also been ascribed to the activation of phosphotyrosine kinases (PTKs) or the inhibition of phosphotyrosine phosphatases (PTPs) in vitro studies [21,24,41]. Results obtained in this paper indicated that oxidative stress-induced tyrosine phosphorylation of certain receptors and substrates may be due to a partial impairment of membrane-bound PTP activity during exhaustive exercise.

PTPs are integral components of signal transduction pathways, and are involved in the control of a variety of cellular tyrosine kinases, such as receptor kinases [42]. PTP is associated with band 3 in the normal human erythrocyte, as shown by co-precipitation of band 3 when PTP is immunoprecipitated [43]. We show here that during exhaustive exercise the oxidative stress-induced band 3 phosphorylation is reversed upon the reduction of the disulfides in the cells. Nevertheless, assay of PTP activity in the absence or presence DTT suggested that reducing agents could increase total PTPase activity, indicating that an oxidative damage affects the PTPs during exhaustive exercise, part of which is reversible (in vivo reduction could be sustained by intracellular GSH). These results lend further support to the conclusion that thiol-alteration and inhibition of PTP is responsible for the appearance of phosphorylated band 3.

Previously, it was shown that exercise-induced oxidant stress injury may have significant effects on RBC hemorheology [4]. Decreased RBC deformability has been reported using different exercise protocols and methods to assess RBC deformability $[8,44-46]$. The extramuscular temperature rise, dehydration hemoconcentration, and increments in intracellular $\mathrm{Ca}^{2+}$ resulting from $\beta$-adrenergic stimulation after acute exposure to exhaustive exercise might be responsible for decreased deformability $[47,48]$. The interactions between membrane, peripheral and cytoskeleton proteins are responsible for the maintenance of RBC deformability, and some of these interactions are modulated by protein phosphorylation status $[49,50]$. Furthermore, several studies have demonstrated that interactions between Band-3 and the cytoskeletal spectrin/actin network play an important function in the maintenance of normal RBC shape and membrane structure. EB3 is also a prominent substrate of Ser/Thr kinases and is the major substrate of RBC protein tyrosine-kinases. Ciana et al. showed the regulation of PTP1B on band 3 of RBC during cell ageing [39]. In vitro studies by Philip S. Low et al. showed that tyrosine phosphorylation of band-3 markedly reduces its affinity for ankyrin, leading to release of band-3 from the spectrin/actin membrane skeleton and enhancing the lateral mobility of band-3 in the bilayer [49]. Indeed, ankyrin docks in close proximity to the band-3 cytoplasmic domain tyrosine phosphorylation sites, and non-phosphorylated band-3 appears to be less prone to forming high-molecular-mass aggregates $[21,51,52]$. The elevated phosphorylation of oxidized/oligomeric band-3 accompanied by the appearance of oxidative damage observed in this study supports the hypothesis that, in exhaustive exercise RBCs, a fraction of band 3 molecules are prone to being oxidatively clustered and tyrosine phosphorylated. This result lends further support to our observation of RBC deformability decreased sharply at all sheer stresses tested after exhaustive exercise. The RBCs transit through the capillaries to supply peripheral skeletal muscle and also cardiac areas with oxygen. Decreased deformability thus prevents the cells from responding to the changing demands by easing the passage through 
the vessel, thereby decreasing the oxygen supply during physical exercise and leading to exercise-related diseases.

In this study, MRE did not induce severe oxidative stress or induce significant changes in EB3 expression and tyrosine phosphorylation. Furthermore, erythrocyte deformability slight increases after MRE. These results are in line with a recent report demonstrating that physical exercise positively influences RBC deformability in untrained rodents $[4,53]$. A recent investigation by Frank Suhr et al. indicated that exercise-induced shear stress stimuli activate RBC-NOS via the PI3-kinase/Akt kinase pathway [54]. Activated RBC-NOS generates NO in RBCs, which is mandatory for the beneficial promotion of RBC rheological functions, e.g., deformability [55]. The results provide a potential molecular pathway underlying the regulation of exercise-induced RBC deformability. However, the underlying in vivo mechanisms remains to be fully resolved.

Also, several studies demonstrated that a change in cell volume could function as the driving force for the stimulated phosphorylation and deformation $[8,15]$. Cell shrinkage was also found to precede the induction of band 3 phosphorylation [15]. In our study, although we tried to keep the body weight and avoid serious dehydration by constantly replenish moisture during exhaustive running exercise. However, exhaustive exercise still resulted in a significant change in RDW, which may caused by exhaustive exercise induced senescence, apoptosis and generation of RBC. In addition, the extent of exhaustive exercise induced changes in red blood cell shrinkage is still not clear. Therefore, the change of band 3 phosphorylation and deformation modulated by erythrocyte shrinkage and ageing during exhaustive exercise regimens must be evaluated further.

\section{Conclusion}

In summary, we presented evidence that exhaustive running exercise results in elevated band-3 tyrosine phosphorylation and alters band-3 membrane organization. These observations also suggest that exhaustive running exercise induced oxidative stress is responsible for the increase in RBC band-3 tyrosine phosphorylation by inhibition of PTP. Under exhaustive exercise conditions, elevated band-3 tyrosine phosphorylation is a potential molecular target of decreased RBC deformability in RBCs. These experimental findings reflect an underlying molecular mechanism by which inadequate supplies of blood and oxygen to tissues leads to exercise-related diseases.

\section{Acknowledgements}

Dr. Xiang Wang was supported by grants from the National Natural Science Foundation of China (NSFC 11072275 and 31271229 ).

\section{References}

$\rightarrow 1$ Alipour M, Salehi I, Ghadiri Soufi F: Effect of exercise on diabetes-induced oxidative stress in the rat hippocampus. Iran Red Crescent Me 2012;14:222-228.

2 Fisher-Wellman K, Bloomer RJ: Acute exercise and oxidative stress: A 30 year history. Dyn Med 2009;8:1.

3 Urso ML, Clarkson PM: Oxidative stress, exercise, and antioxidant supplementation. Toxicology 2003;189:41-54.

4 Yalcin 0, Bor-Kucukatay M, Senturk UK, Baskurt OK: Effects of swimming exercise on red blood cell rheology in trained and untrained rats. J Appl Physiol 2000;88:2074-2080.

5 Groussard C, Rannou-Bekono F, Machefer G, Chevanne M, Vincent S, Sergent O, Cillard J, Gratas-Delamarche A: Changes in blood lipid peroxidation markers and antioxidants after a single sprint anaerobic exercise. Eur J Appl Physiol 2003;89:14-20. 
6 Hakim TS, Macek AS: Effect of hypoxia on erythrocyte deformability in different species. Biorheology 1988;25:857-868.

7 Senturk UK, Gunduz F, Kuru O, Aktekin MR, Kipmen D, Yalcin O, Bor-Kucukatay M, Yesilkaya A, Baskurt OK: Exercise-induced oxidative stress affects erythrocytes in sedentary rats but not exercise-trained rats. J Appl Physiol 2001;91:1999-2004.

8 Mao TY, Fu LL, Wang JS: Hypoxic exercise training causes erythrocyte senescence and rheological dysfunction by depressed gardos channel activity. J Appl Physiol 2011;111:382-391.

-9 Zhang D, Kiyatkin A, Bolin JT, Low PS: Crystallographic structure and functional interpretation of the cytoplasmic domain of erythrocyte membrane band 3. Blood 2000;96:2925-2933.

10 Tanner MJ: Band 3 anion exchanger and its involvement in erythrocyte and kidney disorders. Curr Opin Hematol 2002;9:133-139.

11 Campanella ME, Chu H, Low PS: Assembly and regulation of a glycolytic enzyme complex on the human erythrocyte membrane. P Natl Acad Sci US A 2005;102:2402-2407.

12 Suzuki Y, Ohkubo N, Aoto M, Maeda N, Cicha I, Miki T, Mitsuda N: Participation of caspase-3-like protease in oxidation-induced impairment of erythrocyte membrane properties. Biorheology 2007;44:179-190.

13 Harrison ML, Rathinavelu P, Arese P, Geahlen RL, Low PS: Role of band 3 tyrosine phosphorylation in the regulation of erythrocyte glycolysis. J Biol Chem 1991;266:4106-4111.

14 Minetti G, Piccinini G, Balduini C, Seppi C, Brovelli A: Tyrosine phosphorylation of band 3 protein in $\mathrm{Ca}^{2+}$ / a23187-treated human erythrocytes. Biochem J 1996;320:445-450.

15 Minetti G, Seppi C, Ciana A, Balduini C, Low PS, Brovelli A: Characterization of the hypertonically induced tyrosine phosphorylation of erythrocyte band 3. Biochem J 1998;335:305-311.

16 Harrison ML, Isaacson CC, Burg DL, Geahlen RL, Low PS: Phosphorylation of human erythrocyte band 3 by endogenous p72syk. J Biol Chem 1994;269:955-959.

17 Wang CC, Tao M, Wei T, Low PS: Identification of the major casein kinase i phosphorylation sites on erythrocyte band 3. Blood 1997;89:3019-3024.

18 Pantaleo A, Ferru E, Carta F, Mannu F, Giribaldi G, Vono R, Lepedda AJ, Pippia P, Turrini F: Analysis of changes in tyrosine and serine phosphorylation of red cell membrane proteins induced by $\mathrm{p}$. Falciparum growth. Proteomics 2010;10:3469-3479.

19 Minetti G, Ciana A, Balduini C: Differential sorting of tyrosine kinases and phosphotyrosine phosphatases acting on band 3 during vesiculation of human erythrocytes. Biochem J 2004;377:489-497.

20 Zipser Y, Piade A, Barbul A, Korenstein R, Kosower NS: $\mathrm{Ca}^{2+}$ promotes erythrocyte band 3 tyrosine phosphorylation via dissociation of phosphotyrosine phosphatase from band 3. Biochem J 2002;368:137144.

21 Pantaleo A, Ferru E, Giribaldi G, Mannu F, Carta F, Matte A, de Franceschi L, Turrini F: Oxidized and poorly glycosylated band 3 is selectively phosphorylated by syk kinase to form large membrane clusters in normal and g6pd-deficient red blood cells. Biochem J 2009;418:359-367.

22 Terra HT, Saad MJ, Carvalho CR, Vicentin DL, Costa FF, Saad ST: Increased tyrosine phosphorylation of band 3 in hemoglobinopathies. Am J Hematol 1998;58:224-230.

23 Bordin L, Brunati AM, Donella-Deana A, Baggio B, Toninello A, Clari G: Band 3 is an anchor protein and a target for shp-2 tyrosine phosphatase in human erythrocytes. Blood 2002;100:276-282.

24 Bordin L, Zen F, Ion-Popa F, Barbetta M, Baggio B, Clari G: Band 3 tyr-phosphorylation in normal and glucose-6-phospate dehydrogenase-deficient human erythrocytes. Mol Membr Biol 2005;22:411-420.

25 Davies KJ, Quintanilha AT, Brooks GA, Packer L: Free radicals and tissue damage produced by exercise. Biochem Bioph Res Com 1982;107:1198-1205.

-26 Yakubu MT, Akanji MA, Oladiji AT, Adesokan AA: Androgenic potentials of aqueous extract of massularia acuminata (g. Don) bullock ex hoyl. Stem in male wistar rats. J Ethnopharmacol 2008;118:508-513.

-27 Beutler E, Gelbart T: The mechanism of removal of leukocytes by cellulose columns. Blood Cell 1986;12:5764.

28 de Franceschi L, Turrini F, Honczarenko M, Ayi K, Rivera A, Fleming MD, Law T, Mannu F, Kuypers FA, Bast A, van der Vijgh WJ, Brugnara C: In vivo reduction of erythrocyte oxidant stress in a murine model of betathalassemia. Haematologica 2004;89:1287-1298.

29 de Franceschi L, Rouyer-Fessard P, Alper SL, Jouault H, Brugnara C, Beuzard Y: Combination therapy of erythropoietin, hydroxyurea, and clotrimazole in a beta thalassemic mouse: A model for human therapy. Blood 1996;87:1188-1195.

-30 De Franceschi L, Daraio F, Filippini A, Carturan S, Muchitsch EM, Roetto A, Camaschella C: Liver expression of hepcidin and other iron genes in two mouse models of beta-thalassemia. Haematologica 2006;91:13361342 . 
31 Dringen R, Hamprecht B: Glutathione content as an indicator for the presence of metabolic pathways of amino acids in astroglial cultures. J Neurochem 1996;67:1375-1382.

-32 Misra HP, Fridovich I: The role of superoxide anion in the autoxidation of epinephrine and a simple assay for superoxide dismutase. J Biol Chem 1972;247:3170-3175.

-33 Stocks J, Dormandy TL: The autoxidation of human red cell lipids induced by hydrogen peroxide. Brit J Haematol 1971;20:95-111.

34 Anderson ME: Determination of glutathione and glutathione disulfide in biological samples. Method Enzymol 1985;113:548-555.

-35 Yamaguchi T, Murata Y, Kobayashi J, Kimoto E: Effects of chemical modification of membrane thiol groups on hemolysis of human erythrocytes under hydrostatic pressure. Biochim Biophys Acta 1994;1195:205210.

-36 Dempsey JA, Wagner PD: Exercise-induced arterial hypoxemia. J Appl Physiol 1999;87:1997-2006.

-37 Hopkins SR: Exercise induced arterial hypoxemia: The role of ventilation-perfusion inequality and pulmonary diffusion limitation. Adv Exp Med Biol 2006;588:17-30.

-38 Barbul A, Zipser Y, Nachles A, Korenstein R: Deoxygenation and elevation of intracellular magnesium induce tyrosine phosphorylation of band 3 in human erythrocytes. FEBS letters 1999;455:87-91.

39 Ciana A, Minetti G, Balduini C: Phosphotyrosine phosphatases acting on band 3 in human erythrocytes of different age: Ptp1b processing during cell ageing. Bioelectrochemistry 2004;62:169-173.

-40 Zipser Y, Piade A, Kosower NS: Erythrocyte thiol status regulates band 3 phosphotyrosine level via oxidation/reduction of band 3-associated phosphotyrosine phosphatase. FEBS letters 1997;406:126-130.

41 Brunati AM, Bordin L, Clari G, James P, Quadroni M, Baritono E, Pinna LA, Donella-Deana A: Sequential phosphorylation of protein band 3 by syk and lyn tyrosine kinases in intact human erythrocytes: Identification of primary and secondary phosphorylation sites. Blood 2000;96:1550-1557.

42 Ostman A, Bohmer FD: Regulation of receptor tyrosine kinase signaling by protein tyrosine phosphatases. Trends Cell Biol 2001;11:258-266.

43 Zipser Y, Kosower NS: Phosphotyrosine phosphatase associated with band 3 protein in the human erythrocyte membrane. Biochem J 1996;314:881-887.

-44 Senturk UK, Yalcin O, Gunduz F, Kuru O, Meiselman HJ, Baskurt OK: Effect of antioxidant vitamin treatment on the time course of hematological and hemorheological alterations after an exhausting exercise episode in human subjects. J Appl Physiol 2005;98:1272-1279.

45 Yalcin 0, Erman A, Muratli S, Bor-Kucukatay M, Baskurt OK: Time course of hemorheological alterations after heavy anaerobic exercise in untrained human subjects. J Appl Physiol 2003;94:997-1002.

46 Condon MR, Kim JE, Deitch EA, Machiedo GW, Spolarics Z: Appearance of an erythrocyte population with decreased deformability and hemoglobin content following sepsis. Am J Physiol Heart Circ Physiol 2003;284:H2177-2184.

47 Smith JA: Exercise, training and red blood cell turnover. Sports Med 1995;19:9-31.

48 Szygula Z: Erythrocytic system under the influence of physical exercise and training. Sports Med 1990;10:181-197.

-49 Ferru E, Giger K, Pantaleo A, Campanella E, Grey J, Ritchie K, Vono R, Turrini F, Low PS: Regulation of membrane-cytoskeletal interactions by tyrosine phosphorylation of erythrocyte band 3. Blood 2011;117:5998-6006.

50 Bruce LJ, Beckmann R, Ribeiro ML, Peters LL, Chasis JA, Delaunay J, Mohandas N, Anstee DJ, Tanner MJ: A band 3-based macrocomplex of integral and peripheral proteins in the rbc membrane. Blood 2003;101:4180-4188.

51 Chang SH, Low PS: Identification of a critical ankyrin-binding loop on the cytoplasmic domain of erythrocyte membrane band 3 by crystal structure analysis and site-directed mutagenesis. J Biol Chem 2003;278:6879-6884.

52 Bordin L, Quartesan S, Zen F, Vianello F, Clari G: Band 3 tyr-phosphorylation in human erythrocytes from non-pregnant and pregnant women. Biochim Biophys Acta 2006;1758:611-619.

53 Wang X, Wu Z, Song G, Wang H, Long M, Cai S: Effects of oxidative damage of membrane protein thiol groups on erythrocyte membrane viscoelasticities. Clin Hemorheol Micro 1999;21:137-146.

54 Suhr F, Brenig J, Muller R, Behrens H, Bloch W, Grau M: Moderate exercise promotes human rbc-nos activity, no production and deformability through akt kinase pathway. PloS one 2012; 7:e45982.

55 Bor-Kucukatay M, Wenby RB, Meiselman HJ, Baskurt OK: Effects of nitric oxide on red blood cell deformability. Am J Physiol Heart Circ Physiol 2003;284:H1577-1584. 